Illinois State University

ISU ReD: Research and eData

Theses and Dissertations

7-10-2020

\title{
A Study Of Covid-19 Mortality Under Varying Patient Frailty
}

Alison N. Sifuentes

Illinois State University, alysifuentes@gmail.com

Follow this and additional works at: https://ir.library.illinoisstate.edu/etd

Part of the Epidemiology Commons

\section{Recommended Citation}

Sifuentes, Alison N., "A Study Of Covid-19 Mortality Under Varying Patient Frailty" (2020). Theses and Dissertations. 1310.

https://ir.library.illinoisstate.edu/etd/1310

This Thesis is brought to you for free and open access by ISU ReD: Research and eData. It has been accepted for inclusion in Theses and Dissertations by an authorized administrator of ISU ReD: Research and eData. For more information, please contact ISUReD@ilstu.edu. 


\title{
A STUDY OF COVID-19 MORTALITY UNDER VARYING PATIENT FRAILTY
}

\author{
ALISON N. SIFUENTES
}

33 Pages

Modeled the spread and mortality of COVID-19 throughout the city of Chicago. By incorporating group frailty into a classic SEIR infectious disease model, we were able to differentiate the population of Chicago by their response to COVID-19. Three age groups with different COVID-19-induced death rates were examined, and the model sought to showcase the multiplicative deviation of each age group death rate from the average disease-induced death rate. This adjustment for different death rates among age groups accounted for heterogeneity within the population, and sought to introduce a more accurate manner for modeling the spread of infectious diseases.

KEYWORDS: Ageing, COVID-19, Frailty, Infectious disease modeling, SEIR 


\title{
A STUDY OF COVID-19 MORTALITY UNDER VARYING PATIENT FRAILTY
}

\author{
ALISON N. SIFUENTES
}

A Thesis Submitted in Partial Fulfillment of the Requirements for the Degree of

MASTER OF SCIENCE

Department of Mathematics

\section{ILLINOIS STATE UNIVERSITY}


(c) 2020 Alison N. Sifuentes 


\title{
A STUDY OF COVID-19 MORTALITY UNDER VARYING PATIENT FRAILTY
}

\author{
ALISON N. SIFUENTES
}

COMMITTEE MEMBERS:

Olcay Akman, Chair

Dan Hrozencik

Epaminondas Rosa

Ranee Thiagarajah 


\section{CONTENTS}

Page

CONTENTS

TABLES

FIGURES

CHAPTER I: INTRODUCTION 1

$\begin{array}{ll}\text { CHAPTER II: METHODS } & 10\end{array}$

II.1 Collecting Data 10

II.1.1 Organizing Population Data Collected from the City of $\begin{array}{ll}\text { Chicago } & 10\end{array}$

II.2 Data Exploration 10

II.2.1 Combining Age Group Data for Frailty Estimation 10

II.3 Estimating Frailty Using Age Grouped Data 15

$\begin{array}{ll}\text { CHAPTER III: MODEL } & 17\end{array}$

III.1 Developing a Meta-population SEIR Model 17

$\begin{array}{ll}\text { III.1.1 The Basics } & 17\end{array}$

III.1.2 Introducing Subclasses 18

III.1.3 Introducing Frailty 20

CHAPTER IV: RESULTS 22

CHAPTER V: CONCLUDING REMARKS 30

REFERENCES 


\section{TABLES}

Table $\quad$ Page

1 Covid-19 Age-Stratified Confirmed Cases, Number of Deaths 10

2 Total Deaths and Cases for Age Group Data 15

3 Frailty Parameter Estimation 16

$4 \quad$ Initial Conditions 22

$5 \quad$ Parameter Estimations 23

6 Percent Error for the Number of Deaths 28 


\section{FIGURES}

Figure

Page

1 Compartmental model with three classes: S, I, and R. The parameters $\beta$ and $\gamma$ each represent flow rates between classes. In this diagram, $\beta$ is the rate at which the susceptible become infected and $\gamma$ is the rate at which the infected recover.

2 Compartmental model with four classes: S, E, I, and R. The parameters $\beta, \zeta$, and $\gamma$ each represent flow rates between classes. In this diagram, $\beta$ is the rate at which the susceptible become infected, $\zeta$ is the rate at which the exposed move into the infectious class, and $\gamma$ is the rate at which the infected are removed from the infectious class.

$3 \quad$ Age group total confirmed cases for COVID-19 as of July 5, 2020.

4 Age group total deaths for COVID-19 as of July 5, 2020.

5 Age group death rates for COVID-19 as of July 5, 2020. Deaths rates are calculated per 100,000 people.

6 Age group COVID-19 confirmed cases, deaths, and death rates as of July 5th, 2020.

6 Age group COVID-19 confirmed cases, deaths, and death rates as of July 5th, 2020 .

$7 \quad$ SEIR model with subclasses subdividing the infectious class.

8 SEIR epidemic, where the size of the susceptible, exposed, infectious, and recovered classes are displayed against time. Note that the size of the infectious subclasses is small compared to the size of the total population, so it difficult to examine the change in size for these subclasses with a full model plot.

$9 \quad$ Number of daily COVID-19 confirmed cases for each age group. 
10 Size of the infectious class for each age group. The size of the infectious class is depicted as a proportion of the entire Chicago population. Age Group 1, Age Group 2, and Age Group 3 refer to Infectious Class 1, Infectious Class 2, and Infectious Class 3 respectively.

11 Number of daily COVID-19-induced deaths for each age group.

12 Cumulative COVID-19-induced deaths for each age group when no frailty parameters are included. Cumulative deaths are depicted as proportions of the entire Chicago population.

13 Cumulative COVID-19-induced deaths for each age group when frailty is accounted for. Cumulative deaths are depicted as proportions of the entire Chicago population. 


\section{CHAPTER I: INTRODUCTION}

Infectious disease modeling is used to mimic, analyze, and interpret the spread of infectious diseases through a population. Two common models used for infectious disease spread are compartmental models and agent-based models [1]. Compartmental models organize a population into classes differentiated by health status as directly related to the infectious disease in question $[1,13]$. For example, these models consider everyone who has not contracted the disease to be susceptible, those who have contracted the disease to be infected and arguably infectious, and those who have recovered from the disease (or died from the disease) to be recovered. In this way, compartmental models are able to group individuals within a population into classes based on their disease status. The flow of individuals from one class to another is visually depicted in Fig. (1). In this diagram, individuals flow between the three states: S (Susceptible), I (Infected), and R (Recovered). Each arrow moving between classes is associated with a set of parameters. These parameters showcase the rate at which individuals move out of one class and into the next [13].

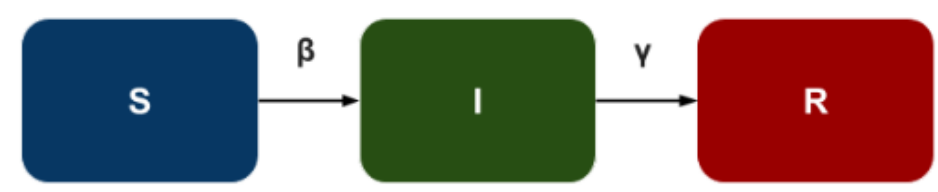

Figure 1: Compartmental model with three classes: $\mathrm{S}, \mathrm{I}$, and $\mathrm{R}$. The parameters $\beta$ and $\gamma$ each represent flow rates between classes. In this diagram, $\beta$ is the rate at which the susceptible become infected and $\gamma$ is the rate at which the infected recover.

For compartmental infectious disease models, it is important to note that all individuals within a population of interest are assumed to fall within one of these classes $[1,13]$. In this way, the model assumes that all susceptible people move into the infected class at the same rate. Similarly, the model assumes that all infected individuals recover at the same rate. Therefore these rates are considered average rates at which individuals move between classes, as the model does not leave room for individualistic choices / health 
/ resources that realistically affect the rate at which individuals become infected and recover. Unlike compartmental models, agent-based models are able to accommodate individualized data such that these models consider the choices / health / resources of each person in the population. Agent-based models however require enormous amounts of data to accurately reflect how individuals within a population interact with one another. To model the spread of infectious diseases without the need for large amounts of data and knowledge pertaining to local movements within a population, compartmental models are typically more widely used. The number of classes that a compartmental model contains, the rates at which each class flows into the next class, and the overall complexity of compartmental models can vary. This variation in compartmental model building provides a flexible and reliable method for analyzing the spread of infectious diseases [1].

One longstanding method for describing and analyzing the spread of infectious disease is the compartmental SEIR model. The SEIR model subdivides the population into four classes: the susceptible, the exposed, the infectious, and the recovered / removed. The SEIR model then models the rate at which people in a population move between these four different states [1]. The diagram in Fig. (2) represents a basic SEIR model with its corresponding flow rates.

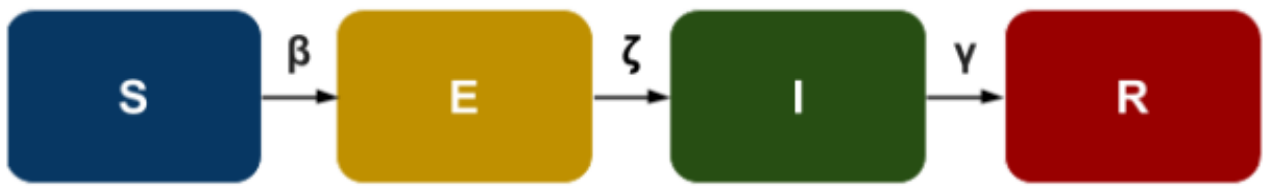

Figure 2: Compartmental model with four classes: S, E, I, and R. The parameters $\beta, \zeta$, and $\gamma$ each represent flow rates between classes. In this diagram, $\beta$ is the rate at which the susceptible become infected, $\zeta$ is the rate at which the exposed move into the infectious class, and $\gamma$ is the rate at which the infected are removed from the infectious class.

For the SEIR model, most individuals begin as susceptible to an infectious disease, and upon interacting with and contracting the disease from infectious individuals, they move into the exposed population [13]. This is illustrated in Fig. (2) between the S 
(Susceptible) and E (Exposed) compartments. The exposed population consists of individuals who have contracted the disease but have yet to show symptoms. Despite the lack of symptoms, it is possible for individuals within the exposed population to spread the disease, as the exposed may become contagious a few days before symptoms start. The specific infectious period, whether beginning before or at the start of symptoms, is dependent on the disease and is often based on disease-specific medical knowledge. Since it is difficult to truly estimate the size of the exposed population that is contagious before symptoms appear, the infectious class is considered to be everyone displaying symptoms and thereby visibly identifiable as infectious. Therefore, those exposed will enter into the infectious population based on whether or not they are symptomatic. This movement is outlined between the E (Exposed) and I (Infectious) compartments in Fig. (2).

Once in the infectious class, individuals can move out of this class upon recovery from the disease, or as a result of mortality - thus moving into the recovered / removed class. The recovered / removed population consists of individuals who have died either due to the disease or other causes, as well as individuals who are immune to contracting the disease after initial survival (this is dependent upon the disease, as there are some diseases where recovery doesn't offer immunity from reinfection). For those diseases offering immunity upon recovery, there will be no arrow stemming from the recovered class to the susceptible class. For those diseases where reinfection is possible, there can be an arrow connecting the recovered class to the susceptible class. Altogether, population movement between the susceptible, exposed, infectious, and recovered / removed classes is dependent on the infectious disease of interest, as flow rates between classes depend on the epidemiological rates predetermined by medical research.

It is important to note that flow rates between classes for an SEIR model treat all individuals as having an averaged susceptibility, exposure, mortality, and recovery response to a given infectious disease. This falls back onto the basic model assumptions of the SEIR model. The model assumes that as individuals move between classes, there is homogeneity 
of individuals as well as spatial-temporal homogeneity $[1,17]$. The focus of this work is to take a closer look at how different age groups within the population respond to the transitions between the S, E, I, and R states. We may assume, for example, that all infectious individuals recover at the same rate. However, due to differing health and immune system factors, not everyone with a given disease will respond in the same manner. The factors influencing individual response to a disease contribute to an individual's "frailty." The term frailty from a medical viewpoint encompasses those attributes or characteristics placing some of the population at an advantage and others at a disadvantage during a disease outbreak [11]. Variation in frailty regarding health status is one way of introducing heterogeneity within a population. The overall health and responsiveness of individuals within a population can shed light on the spread of infectious diseases.

One method for incorporating heterogeneity within an SEIR model is to break down the typical susceptible, exposed, infectious, and recovered / removed classes into subclasses. These subclasses can be defined by epidemiological research differentiating a population by physical attributes such as age, sex, and/or race. Subclasses may also be organized via attributes concerning post traumatic stress disorder (PTSD), access to healthcare, and location. Altogether, subclasses allow for differentiation within a population. For example, instead of assuming that all infectious individuals recover at the same rate, we can break down the infectious class into subclasses distinguished by access to healthcare. The rate at which individuals recover may differ depending on individual access to healthcare and healthcare quality. By dividing the infectious class into subclasses based on healthcare access, the model more accurately depicts the flow of individuals moving between the infectious and recovered / removed classes. In this way, subclasses allow for differentiation within a population containing noticeable clustered responses to an infectious disease. These models containing subclasses to further explain the spread of infectious disease and understand the underlying dynamics occurring within a population are referred to as meta-population models [1]. 
Regarding factors differentiating individuals within a population into subclasses, one can examine group frailty. Frailty, in a medical sense, marks a difference in response due to varying health factors [4]. From a mathematical standpoint, frailty is a multiplicative effect on the hazard function of a group of individuals [15]. Moreover, frailty, in terms of disease modeling, represents a multiplicative effect on the response of individuals who have contracted a disease. Those individuals considered to be more frail, whether due to internal characteristics or external circumstances, undergo a multiplicative effect on their corresponding death rate. This work incorporates the concept of frailty to develop an SEIR meta-population model accounting for heterogeneity within classes, as well as a method for estimating the multiplicative effect distinguishing this heterogeneity as a result of frailty. The multiplicative effect due to frailty is defined as the frailty parameter. To demonstrate one method for estimating the frailty parameters within a meta-population model, we sought to model the spread of the coronavirus COVID-19 throughout the city of Chicago in Illinois.

Coronaviruses are a subset of viruses causing illness in animals and sometimes humans depending on the development of the virus. Coronaviruses can have differing effects on animals and humans if infected, but most commonly these viruses lead to respiratory complications. In humans particularly, these viruses can range in their symptomatic seriousness as someone infected may display typical common cold-like symptoms, while someone else may experience respiratory failure and pneumonia [2]. Different pathogen strains of coronavirus have emerged within the last two decades. Most notably are SARS, MERS, and COVID-19. SARS led to an outbreak in the years 2002-2004, while MERS led to an outbreak in 2012. COVID-19 is the name given to a strain of coronavirus, SARS-CoV-2, leading to the global pandemic in 2020. This virus is an infectious disease spreading from person to person with an infection rate higher than that of SARS and MERS during their respective outbreaks [2]. COVID-19 symptoms range as they do for most strains of coronaviruses, however common symptoms indicating 
infection include a consistent cough, fever, shortness of breath, and body aches. Infected individuals may also experience a sore throat, loss of taste and smell, diarrhea, as well as a recurring headache [3]. Some individuals infected with COVID-19 may develop COVID-related pneumonia, a serious complication in which the air sacs within the lungs become infected. If pneumonia develops, patients may experience a phlegm-filled cough in addition to COVID-19 symptoms [4].

The symptoms experienced by individuals infected by COVID-19 vary in their seriousness and longevity. Some infected individuals may recover quickly and experience mild symptoms while others may take as long as 3-6 weeks to recover from more serious respiratory complications. Although infected individuals of all ages have undergone serious symptoms and required hospitalizations, older populations are considered at high risk for COVID-19 [5]. This risk is attributed to the relationship of age and comorbidities. Comorbidities describe the accumulation of existing factors / conditions that can impact response to an immediate or primary condition, such as a disease. Some conditions occurring together that can be considered comorbidities include hypertension, high cholesterol, diabetes, dementia, and more [6]. Comorbidities are associated with age as the number of comorbidities accumulated increases as age increases [8]. Individuals over the age of 65 are especially associated with having developed more conditions in co-occurrence, thus having developed more comorbidities [8]. These comorbidities lead to complications regarding individual response to diseases such as COVID-19. Since age is associated with development of comorbidities and increased risk factors, age can be considered a factor differentiating a population by their response to a disease, or by their individual frailty.

Alongside comorbidities serving as a link between age and frailty, age has been defined as a frailty itself. A study examining pneumonia risk factors considered age a frailty factor, defining frailty as "a distinct clinical syndrome characterized by a decrease in physiological reserve and resistance to stressful situations, making individuals more vulnerable to health problems" [9]. Another study provides evidence that age increases 
rates of chronic disease and impairments [10]. Whatsmore, one study formally defined aging as "changes in immune response impairment of alveolar macrophage function and increase in cellular apoptosis during sepsis, leading to a greater severity of infection" [11]. This study went on to suggest that "biological age should be more routinely assessed to guide clinical decision making in older patients in general and, in particular, to help clinicians identify older patients with pneumonia who might benefit from ICU admission." While this study focuses on pneumonia rather than COVID-19, pneumonia is a possible complication of COVID-19, and it is important to note the risk associated with age in non-COVID pneumonia patients. This study determined that age was a frailty affecting mortality of pneumonia patients [11].

As a result of this evidence and available data, throughout this work, we consider frailty in the context of age. This means that as we proceed towards accounting for variation due to "frailty," and build upon the compartmental SEIR infectious disease model, we are really examining the effects of aging on patient response to an infectious disease. While frailty can account for any internal or external attribute impacting individual response to a disease, we focus in on aging as a characteristic marker for frailty. However, we continue to refer to variation in response to disease through the general term "frailty" throughout this work as our model and method may be modified to address variation due to any factor affecting frailty. Yet for our specific case study, the term "frailty" is considered interchangeable with ageing.

Since age is considered a frailty, we have decided to differentiate a COVID-19 affected population by age group. The goal is to create a metapopulation model incorporating age group frailty and to use the COVID-19 outbreak as a case study. Since frailty is a multiplicative effect on the hazard function, or death rate, it is of interest to focus the frailty within the model on COVID-19 mortality rates. From a public health standpoint, older individuals are presumed at higher risk for COVID-19, such that they are more likely to undergo worsened symptoms and may require hospitalization [5]. To 
mathematically estimate just how age affects an individual's frailty for COVID-19 mortality, age group death rates were estimated using data collected from the city of Chicago.

The city of Chicago was among the "hot spots" when the COVID-19 outbreak reached the United States. The terminology "hot spot" refers to a location in which the number of cases arose rapidly. Hot spots also refer to locations containing more cases than most other locations in the country at the time. The city of Chicago, as well as the entirety of the state of Illinois, took immediate response to rising case numbers and concerns regarding COVID-19. As a result of measures put in place to limit the spread of COVID-19 throughout Illinois, the number of in-person interactions among Chicago residents was forcibly lessened for the majority of the outbreak. This consistent and immediate response marked Chicago as an adequate place to model. Moreover, the consistency of the Chicago-central outbreak was considered a good measure for meeting the assumptions of the compartmental SEIR model.

The adequacy of compartmental SEIR models depends largely on homogeneous interaction, spatial homogeneity, and temporal homogeneity $[1,13]$. Modeling the entirety of the United States, for example, would break the assumption of homogenous interaction as not everyone in the United States is interacting with an equal number of people each day. Even modeling on a smaller scale, say the entirety of Illinois, breaks the assumption of spatial homogeneity as people living in rural areas theoretically interact with fewer people daily when compared with people living in urban areas. Averaging interaction and infection rates across rural and urban areas may lead to spurious conclusions if left unacknowledged. Chicago, as a centralized and strictly urban location, was considered to be more consistent in its number of daily in-person interactions. In addition, Chicago more closely meets the assumption of spatial homogeneity (as an all urban location) than the state of Illinois or the entirety of the United States. Furthermore, Chicago remained uniform in it's lockdown policy for the majority of the outbreak, only opening up once the number of daily cases 
was lessened. Using the Chicago-central outbreak as a case study, data collection and the method for introducing frailty to different age groups is outlined in the Methods section below. 


\section{CHAPTER II: METHODS}

\section{II.1 Collecting Data}

\section{II.1.1 Organizing Population Data Collected from the City of Chicago Data}

containing daily COVID-19 mortality counts and confirmed COVID-19 case counts was collected from the City of Chicago website and published to HealthData.gov. This data contained the number of COVID-19 deaths as well as confirmed cases for varying demographics within Chicago. More specifically, the dataset included COVID-19 mortality and case counts for eight different age groups: ages younger than 18, ages 18-29, ages 30-39, ages 40-49, ages 50-59, ages 60-69, ages 70-79, and ages 80 and older. As an overview, the total COVID-19 mortality and confirmed case counts for each age group are included below in Table (1).

Table 1: Covid-19 Age-Stratified Confirmed Cases, Number of Deaths

\begin{tabular}{|c|c|c|c|}
\hline Age Group & Confirmed Cases & Number of Deaths & Death Rates \\
\hline$<18$ & 2,636 & 2 & 0.000758725 \\
$18-29$ & 10,090 & 18 & 0.001783944 \\
$30-39$ & 9,521 & 65 & 0.006827014 \\
$40-49$ & 9,612 & 142 & 0.0147732 \\
$50-59$ & 9,024 & 289 & 0.032025709 \\
$60-69$ & 6,400 & 573 & 0.08953125 \\
$70-79$ & 3,477 & 652 & 0.187517975 \\
$80-89$ & 2,751 & 893 & 0.324609233 \\
\hline
\end{tabular}

\section{II.2 Data Exploration}

II.2.1 Combining Age Group Data for Frailty Estimation The data in Table (1). provides the total number of COVID-induced deaths and confirmed COVID-19 cases for eight age groups. When investigating differences in frailty, it is not especially important to look at each of these particular age groups separately. Instead, we want to focus on groups displaying obvious differences in the number of deaths per the corresponding number of confirmed cases. Examining a bar graph of the number of confirmed cases per age group, 
shown in Fig. (3), notice that the majority of COVID-19 cases are among age groups 2-5, corresponding to ages 18-59. Interestingly, despite there being more cases within these age groups, age groups 2-5 have fewer total deaths than age groups 6-8. A bar graph for age-stratified total COVID-19 deaths is provided in Fig. (4).

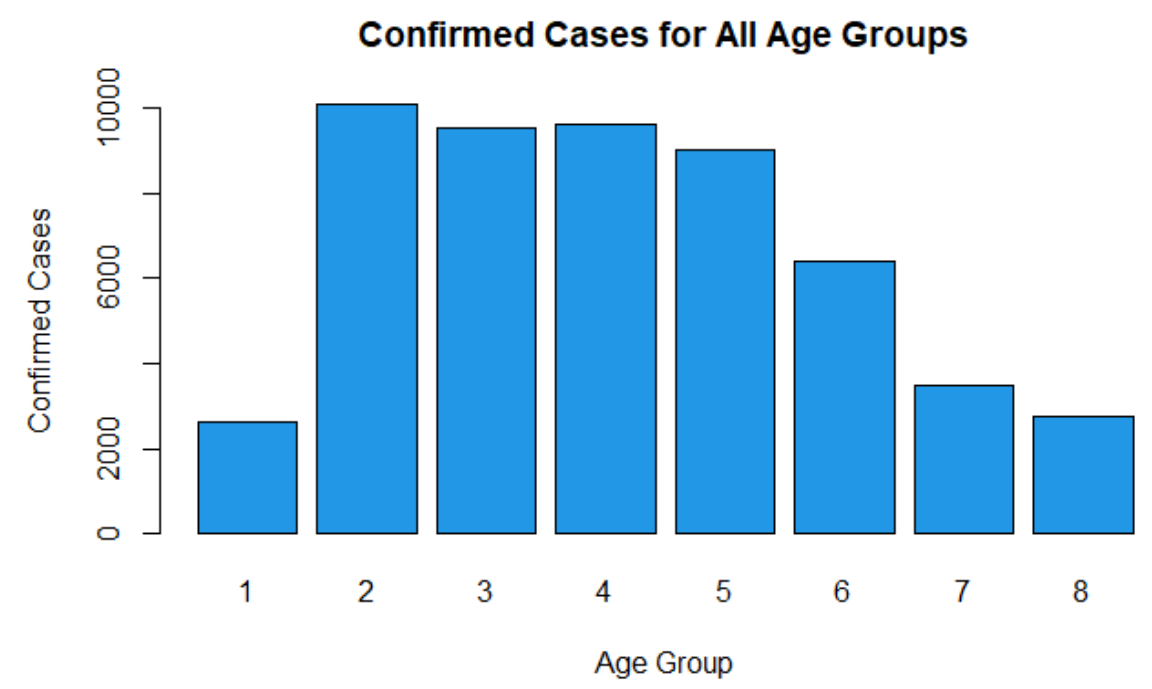

Figure 3: Age group total confirmed cases for COVID-19 as of July 5, 2020.

To gain a visual perspective on how the number of deaths within each age group compares to the corresponding number of cases, age group death rates were plotted in Fig. $(5)$. 


\section{Total Deaths for All Age Groups}

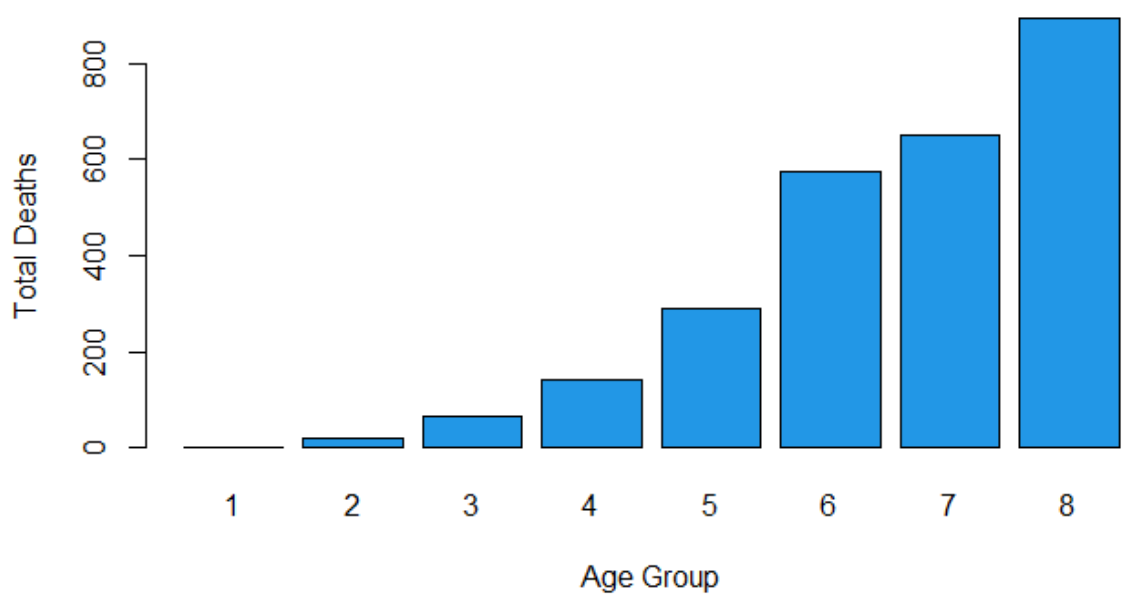

Figure 4: Age group total deaths for COVID-19 as of July 5, 2020.

Death Rates per 100,000 People for All Age Groups

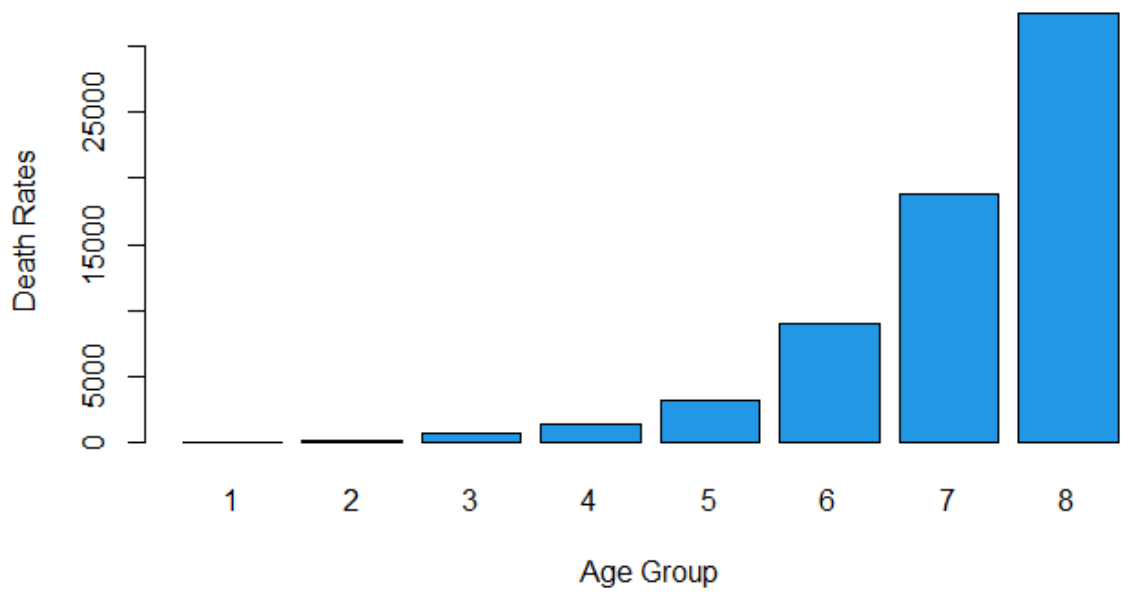

Figure 5: Age group death rates for COVID-19 as of July 5, 2020. Deaths rates are calculated per 100,000 people.

The overall shape of the bar graph for age group death rates is similar to the overall shape of the bar graph for age group deaths. The shape of the bar graph for age group confirmed cases, however, is not similar to that of the graph for deaths and death rates. This indicates that the proportion of deaths to the number of cases is not the same for 
each age group. These proportional differences reflect the difference in response to COVID-19, such that not all age groups respond the same. Since age groups 6-8 maintained high death rates while age groups 2-5 had lower death rates, these clusters of age groups were each grouped together into two separate groups. Furthermore, the youngest age group within the dataset was included as a separate third group. This grouping was completed to showcase noticeable variation in response to COVID-19. If the original eight age groups collected from the city of Chicago dataset were each utilized for comparison, we would expect that some age groups, such as age groups 2-5, would have similar estimates for frailty. Therefore, we would expect those similar age groups to have similar estimations for the number of cumulative deaths. To avoid having excess similar groups, and to focus on the variation as a result of age, the data was reorganized to display three age groups rather than eight. These three age groups contained individuals less than 18 years old, individuals between 18-59 years old, and individuals 60 years old and older. These stratified age groups are in accordance with public health advice differentiating older age groups as being more at risk. Bar graphs for total confirmed cases, total deaths, and death rates for the organized three age groups are outlined in Fig. (6). 


\section{Confirmed Cases for Age Groups}

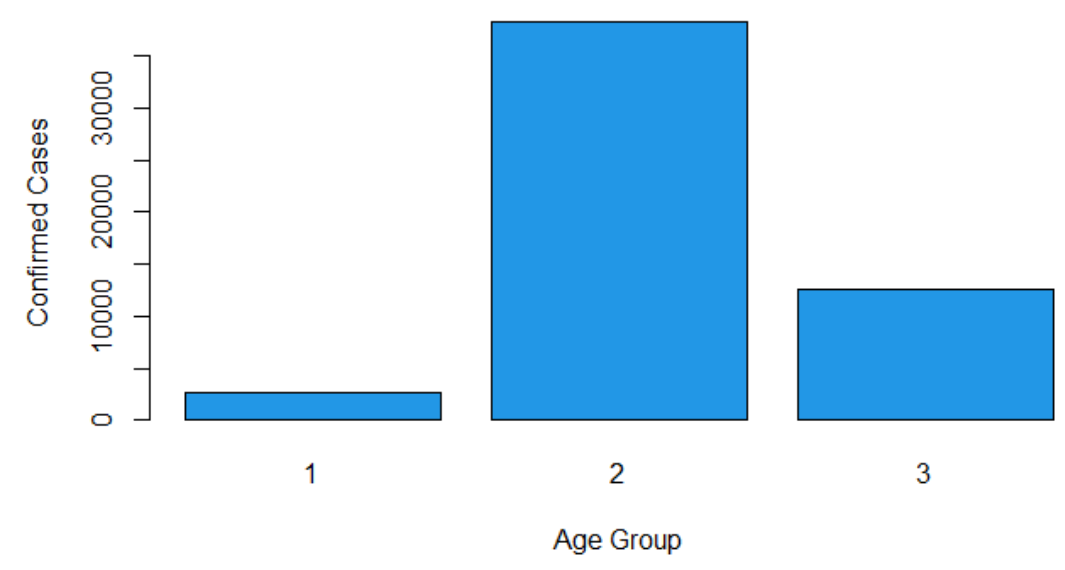

(a) Age group total confirmed cases as of July 5th, 2020.

Total Deaths for Age Groups

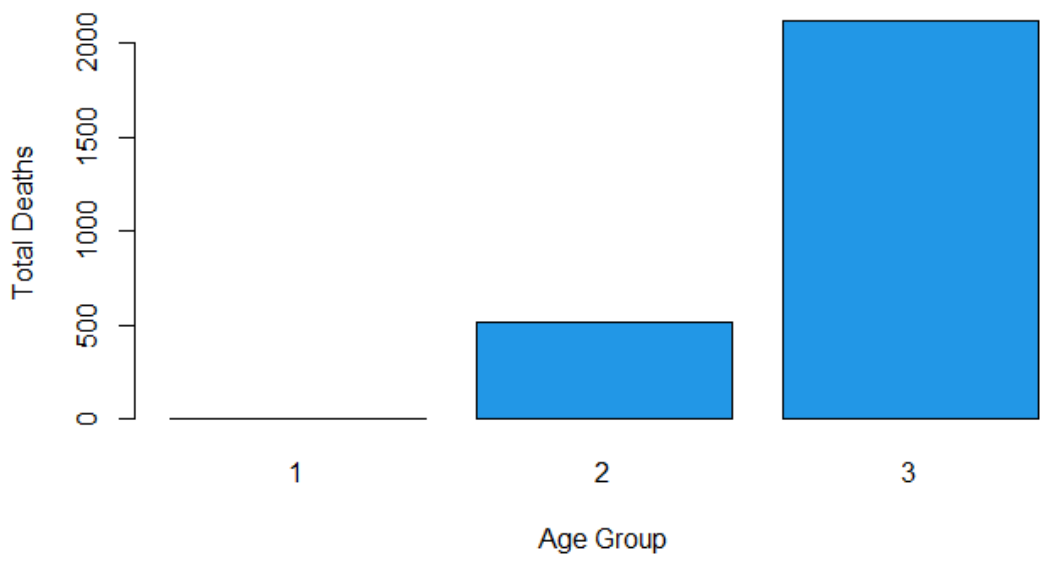

(b) Age group total deaths as of July 5, 2020.

Figure 6: Age group COVID-19 confirmed cases, deaths, and death rates as of July 5th, 2020 .

These three groups exemplify the difference in COVID-19 death rates among age groups. The death rate for the eldest age group (ages 60 years and older) appears much higher from a visual standpoint. To examine just how much more at risk older groups are, we used the data for all three age groups to estimate the frailty for each group. The frailty for each group indicates the multiplicative deviation from the average death rate, thereby showcasing the higher or lower mortality rates experienced by different age groups. 
Death Rates per 100,000 People for Age Groups

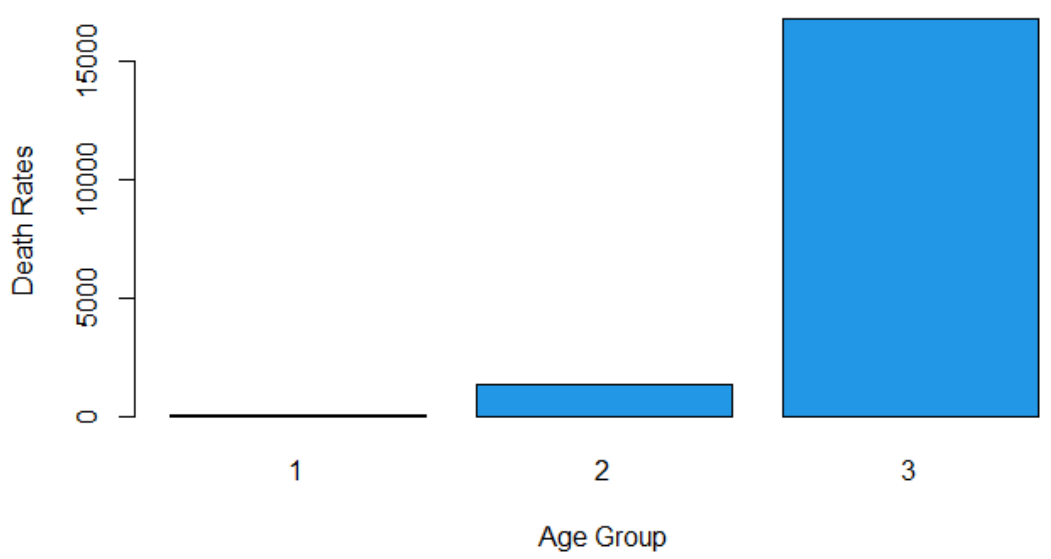

(c) Age group death rates per 100,000 people as of July 5, 2020.

Figure 6: Age group COVID-19 confirmed cases, deaths, and death rates as of July 5th, 2020.

\section{II.3 Estimating Frailty Using Age Grouped Data}

Frailty is a multiplicative effect on the death rate that differentiates a population by some characteristic putting some individuals at an advantage and others at a disadvantage. More specifically, the frailty parameter is an estimate for the multiplicative deviation of differentiated group death rates from the overall average death rate. The average death rate stems from the total number of deaths out of the size of the population at risk (in our case Chicago). Therefore, when calculating the frailty parameter, we find the multiplicative deviation of each group death rate from the overall death rate calculated using the data at hand.

Table 2: Total Deaths and Cases for Age Group Data

\begin{tabular}{|c|c|c|c|c|}
\hline Age & Total Deaths & Population Size & Deaths / Pop. & Group Proportion \\
\hline$<18$ & 2 & 576,625 & 0.000003468 & 0.212107167 \\
$18-59$ & 514 & $1,676,504$ & 0.000306590 & 0.616689381 \\
$60+$ & 2,118 & 465,426 & 0.004550670 & 0.171203452 \\
All & 2,634 & $2,718,555$ & 0.000968897 & 1.0 \\
\hline
\end{tabular}


We find the average death rate by first calculating the number of deaths divided by the size of the population. According to 2020 estimates, the estimated population size of Chicago was $2,718,555$. Thereby, the average death rate is 2,634 divided by $2,718,555$. This gives an average death rate of 0.000969 , or 96.89 deaths per 100,000 people. If there was no frailty, this death rate is the average expected death rate per each age group. However, since some age groups are advantageous in their response to COVID-19 while others are at a disadvantage, this weighted average death rate is not the reality for each age group. Frailty parameters are then used to indicate each age group's deviation from the average death rate. These parameters are estimated by finding the multiplicative deviation of each age-specific death rate from the weighted average death rate. Frailty parameter estimation is outlined below.

Table 3: Frailty Parameter Estimation

\begin{tabular}{|c|c|c|c|}
\hline Age & Age-Specific Death Rate & Average Death Rate & Frailty Parameter \\
\hline$<18$ & 0.000003468 & 0.000968897 & 0.003579801 \\
$18-59$ & 0.000306590 & 0.000968897 & 0.316432351 \\
$60+$ & 0.004550670 & 0.000968897 & 4.696752426 \\
\hline
\end{tabular}

These three frailty parameter estimates - 0.00357980, 0.316432351, and 4.696752426 - are the multiplicative factors showcasing each age group's deviation from the average death rate. Using each frailty parameter, the goal was to more accurately reflect age-stratified mortality for COVID-19 and build a mathematical method of frailty into the classic SEIR infectious disease model. This method sought to tie together medical knowledge of frailty with disease spread. To investigate the adequacy of including these frailty parameters, we built an SEIR model with incorporated frailty. 


\section{CHAPTER III: MODEL}

\section{III.1 Developing a Meta-Population SEIR Model}

III.1.1 The Basics To develop a meta-population SEIR model, the typical SEIR model was first examined as a base for building upon. This basic preliminary SEIR model is included below.

$$
\begin{gathered}
\frac{d S}{d t}=-\beta S(I / N) \\
\frac{d E}{d t}=\beta S(I / N)-\zeta E \\
\frac{d I}{d t}=\zeta E-\gamma I-\delta I \\
\frac{d R}{d t}=\gamma I
\end{gathered}
$$

In Eqs. (1), $\beta$ is the rate at which susceptibles and the infectious interact and at which the disease is spread, $\zeta$ is the rate at which the exposed move into the infectious class, $\gamma$ is the rate at which the infectious move into the recovered class, and $\delta$ is the disease-induced death rate. The parameter $\mathrm{N}$ is also included to represent the total size of the population being examined. Thereby, $\mathrm{N}$ is the sum of each class, or $\mathrm{N}=\mathrm{S}+\mathrm{E}+\mathrm{I}+\mathrm{R}$. We divide the infectious class by $\mathrm{N}$ for Eq. (1.1) and Eq (1.2) to showcase the proportion of infectious people within the population interacting with the susceptible. The parameters $\beta$ and $\delta$ are calculated based on population dynamics and collected data, whereas the parameters $\zeta$ and $\gamma$ stem from medical knowledge regarding the disease. More specifically, $\zeta$ is the reciprocal of the average latent period of a disease, and $\gamma$ is the reciprocal of the average infectious period of a disease multiplied by the percent of recoveries $[16,18]$. It is important to note that the flow rates between the susceptible and infectious classes are based on horizontal incidence (where $\beta$ represents the interaction between the susceptible and infectious) $[16,18]$. Furthermore, the flow rates between the exposed to infectious class and the 
infectious to recovered class are based on the transfer rate of individuals between classes [16]. This means that $\zeta$ and $\gamma$ are the rates at which people move out of the class. Note that this base model does not include a birth rate or death rate so that the total population size is assumed to be constant. While Eqs. (1) demonstrates the flow of individuals between classes, the goal was to create a meta-population model such that at least one class contains subclasses serving to introduce controlled heterogeneity into the model. As a result, the base model was built upon by creating subclasses for each of the age groups within Table 1.

III.1.2 Introducing Subclasses There are three age groups in Table 1: ages less than 18, between 18-59, and greater than 60 . Since frailty is an effect on the disease-induced mortality rate for different age groups, subclasses were constructed within the infectious and recovered classes of the SEIR model. The assumption here is that everyone is equally susceptible to contracting COVID-19, and since the rate at which exposed individuals move into the infectious class is based on the latent period of the disease, it is additionally assumed that the rate $\zeta$ is the same for all age groups. In terms of frailty, some individuals may be at an advantage and others at a disadvantage once they've contracted COVID-19 and their body is battling symptoms. Therefore the difference in frailty is incorporated into the infectious class where individuals either recover or die as they are battling the disease. As a result, these differences in response to the disease are showcased by creating subclasses within the infectious class of the SEIR model and altering the disease-induced death rate. These subclasses are visually depicted in Fig. (7). 


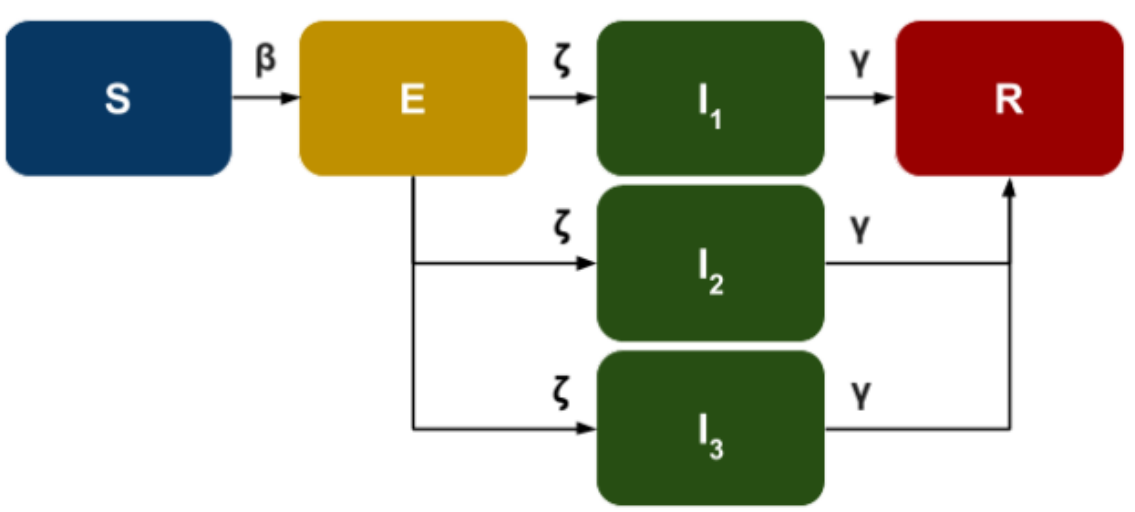

Figure 7: SEIR model with subclasses subdividing the infectious class.

Each of the infectious subclasses, labeled as $\mathrm{I}_{1}, \mathrm{I}_{2}$, and $\mathrm{I}_{3}$, mark a differentiation in the population. The frailty will be introduced regarding each infectious class's COVID-19-induced death rate. For now, this intermediate model with subclasses is represented using the system of differential equations in Eqs. (2).

$$
\begin{gathered}
\frac{d S}{d t}=-\beta S\left(I_{1}+I_{2}+I_{3}\right) / N \\
\frac{d E}{d t}=\beta S\left(I_{1}+I_{2}+I_{3}\right) / N-\zeta E \\
\frac{d I_{1}}{d t}=p_{1} \zeta E-\gamma I_{1}-\delta I_{1} \\
\frac{d I_{2}}{d t}=p_{2} \zeta E-\gamma I_{2}-\delta I_{2} \\
\frac{d I_{3}}{d t}=p_{3} \zeta E-\gamma I_{3}-\delta I_{3} \\
\frac{d R}{d t}=\gamma I_{1}+\gamma I_{2}+\gamma I_{3}
\end{gathered}
$$

Proportions of the population in the form of $\mathrm{p}_{1}, \mathrm{p}_{2}$, and $\mathrm{p}_{3}$ were included in each infectious subclass equation to reflect the number of people in each age group. These proportions were collected by dividing the number of people per age group by the total number of 
people within the population. If these proportions had not been included, then the model would assume that all Exposed move into $\mathrm{I}_{1}, \mathrm{I}_{2}$, and $\mathrm{I}_{3}$. Instead, we need for those exposed to move only into one of those three classes, so the proportions dependent on age group are used to ensure not all exposed move into each infectious subclass.

III.1.3 Introducing Frailty While Eqs. (2) creates three subclasses within the Infectious class, it does not differentiate mortality rates between the three classes. To differentiate mortality rates and account for differences in response to COVID-19, the frailty parameters were introduced. These parameters are labeled as $\phi_{1}, \phi_{2}$, and $\phi_{3}$, such that they correspond to each age group. Since frailty is a multiplicative effect on the death rate, the overall COVID-19 death rate $\delta$ was left in the model, and each of the frailty parameters $\phi_{\mathrm{i}}$, where $\mathrm{i}=1,2,3$, were introduced as multiplicative factors on $\delta$. This was intended to showcase the average death rate and the deviation of each age group from this average as a result of differing frailties. The final model with frailty parameters $\phi_{\mathrm{i}}$ is shown below in Eqs. (3).

$$
\begin{gathered}
\frac{d S}{d t}=\lambda-\beta S\left(I_{1}+I_{2}+I_{3}\right) / N-\mu S \\
\frac{d E}{d t}=\beta S\left(I_{1}+I_{2}+I_{3}\right) / N-\left(\zeta_{1}+\mu\right) E \\
\frac{d I_{1}}{d t}=\zeta E-\left(\gamma+\mu+\phi_{1} \delta\right) I_{1} \\
\frac{d I_{2}}{d t}=\zeta E-\left(\gamma+\mu+\phi_{2} \delta\right) I_{2} \\
\frac{d I_{3}}{d t}=\zeta E-\left(\gamma+\mu+\phi_{3} \delta\right) I_{3} \\
\frac{d R}{d t}=\gamma I_{1}+\gamma I_{2}+\gamma I_{3}-\mu R \\
\frac{d D_{1}}{d t}=\phi_{1} \delta I_{1} \\
\frac{d D_{2}}{d t}=\phi_{2} \delta I_{2}
\end{gathered}
$$




$$
\frac{d D_{3}}{d t}=\phi_{3} \delta I_{3}
$$

The model in Eqs. (3) includes the frailty parameters $\phi_{\mathrm{i}}$ as well as parameters for the natural introduction and removal of individuals to and from the total population. The parameter $\lambda$ represents the population birth rate and the parameter $\mu$ represents the non-COVID-death rate. [13] The parameters $\lambda$ and $\mu$ enable for the size of the total population to grow and diminish from natural causes, such that the disease-induced death rate is not the only way in which people leave the population. These parameters for introducing and removing people to and from a population are important if an outbreak is long-lasting and natural births and deaths would greatly effect the results. If an outbreak occurs more quickly, it is not always necessary for these parameters to be estimated. We leave these parameters as a part of the finalized model so that the model itself can be flexible for different outbreak lengths. While natural birth and death occurrences are considered within the model, the model does not allow for immigration and emigration. Additionally, equations $\mathrm{D}_{1}, \mathrm{D}_{2}, \mathrm{D}_{3}$ were included to examine the cumulative number of disease-induced deaths per each age group. These equations are focused on the number of COVID-19 induced deaths within their corresponding infectious subclass. This was the final model constructed to be used for analysis. 


\section{CHAPTER IV: RESULTS}

To examine how adequately this model reflects the number of deaths within each age group, the software program $\mathrm{R}$ was utilized. Initial conditions for each class size were originally input as whole numbers of the entire city of Chicago population. These class sizes were then scaled to be proportions of the entire population for simpler interpretation. Note that $\mathrm{N}=\mathrm{S}+\mathrm{E}+\mathrm{I}_{1}+\mathrm{I}_{2}+\mathrm{I}_{3}+\mathrm{R}+\mathrm{D}_{1}+\mathrm{D}_{2}+\mathrm{D}_{3}$, where $\mathrm{N}$ is the total size of the population. For studying the spread of COVID-19 in Chicago, the total population is the estimated Chicago population size of 2,718,555 people. Using this total population size, initial conditions were set as outlined below in Table (4).

Table 4: Initial Conditions

\begin{tabular}{|c|c|c|}
\hline Class & Initial Values & Proportion \\
\hline $\mathrm{S}$ & $2,718,555$ & 1 \\
$\mathrm{E}$ & 40 & $40 / 2,718,555$ \\
$\mathrm{I}_{1}$ & 10 & $10 / 2,718,555$ \\
$\mathrm{I}_{2}$ & 10 & $10 / 2,718,555$ \\
$\mathrm{I}_{3}$ & 10 & $10 / 2,718,555$ \\
$\mathrm{R}$ & 0 & 0 \\
$\mathrm{D}_{1}$ & 0 & 0 \\
$\mathrm{D}_{2}$ & 0 & 0 \\
$\mathrm{D}_{3}$ & 0 & 0 \\
\hline
\end{tabular}

Parameter estimations were calculated using the data collected from the City of Chicago, as well as medical knowledge regarding how COVID-19 spreads. Please note that the average COVID-19-induced death rate was more recently estimated at about 0.5 percent [14]. This estimate was used to calculate the disease-induced death rate. The resulting parameter estimations are depicted below in Table (5).

Using these parameter estimations, the model was run using the language $\mathrm{R}$ for a total of 1,500 time steps, where each time step is set as one tenth of a day. A series of graphs were output within $\mathrm{R}$ for referencing the change in size of each class over time. The size of the susceptible, exposed, infectious, and the recovered classes are illustrated in Fig. 
Table 5: Parameter Estimations

\begin{tabular}{|c|c|c|}
\hline Parameter & Description & Estimate \\
\hline$\lambda$ & Natural birth rate & 0.0 \\
$\beta$ & Interaction rate & 0.115 \\
$\mu$ & Natural death rate & 0.0 \\
$\zeta$ & Exposed becoming infectious & 0.2 \\
$\gamma$ & Recovery rate & $0.1 * 0.995$ \\
$\delta$ & Covid-induced death rate & $0.1 * 0.005$ \\
$\phi_{1}$ & Frailty parameter for group 1 & 0.003579801 \\
$\phi_{2}$ & Frailty parameter for group 2 & 0.316432351 \\
$\phi_{3}$ & Frailty parameter for group 3 & 4.696752426 \\
\hline
\end{tabular}

(8).

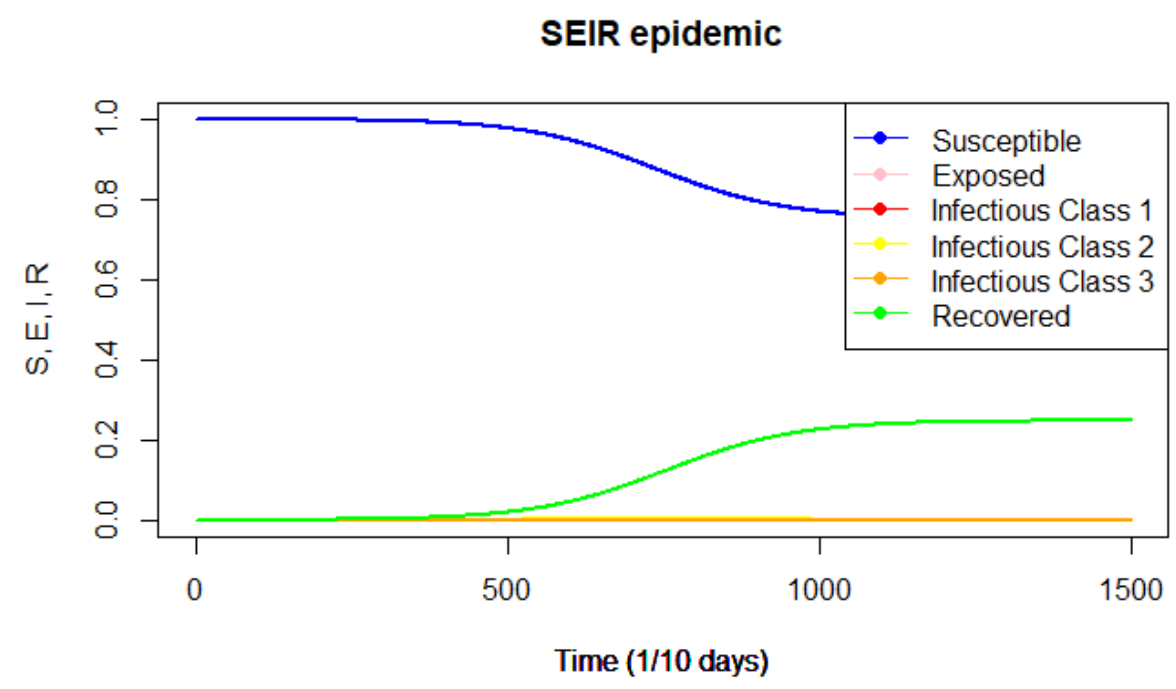

Figure 8: SEIR epidemic, where the size of the susceptible, exposed, infectious, and recovered classes are displayed against time. Note that the size of the infectious subclasses is small compared to the size of the total population, so it difficult to examine the change in size for these subclasses with a full model plot.

It is difficult, however, to examine how the size of each infectious subclass changes over time when viewing a complete model of all classes on the same plot. This is because the overall size of each infectious class is small compared to the total population size of Chicago, and thus this portion of the graph remains small. We can, however, examine the 
change in size of the susceptible and recovered classes within Fig. (8). While the size of the susceptible class decreases over time as more of the susceptible population becomes infected, the recovered class increases over time and grows most rapidly during the peak for each infectious subclass. As the size of the infectious subclasses decreases, the number of recovered individuals begins to increase at a slower rate and eventually plateaus as the outbreak comes to an end. Since it is difficult to examine the outbreak for the full model plot regarding the number of people who become infected, the infectious subclasses were graphed separately from the entire model. In addition to this plot, a plot of the total confirmed COVID-19 cases was included for comparison.

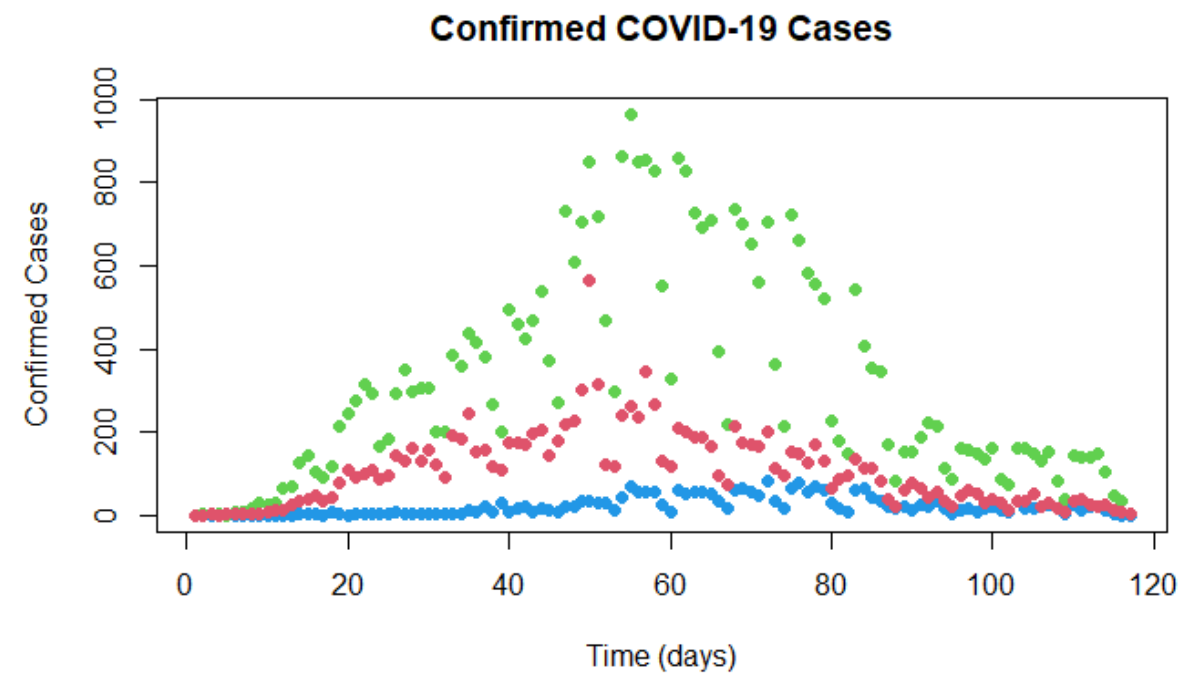

Figure 9: Number of daily COVID-19 confirmed cases for each age group. 
Size of Infectious Classes

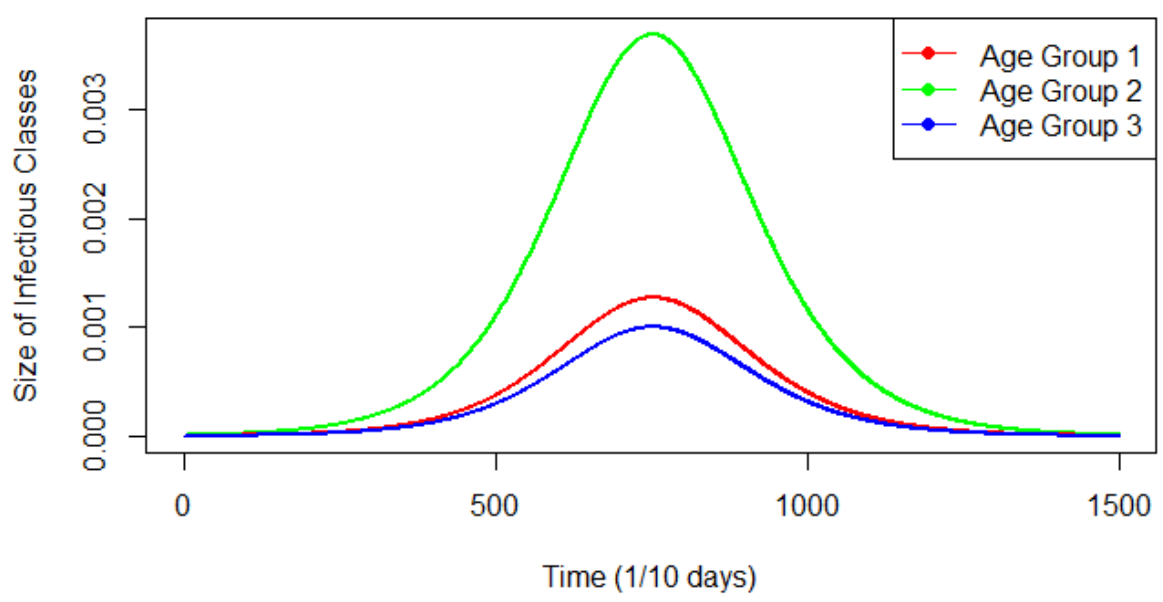

Figure 10: Size of the infectious class for each age group. The size of the infectious class is depicted as a proportion of the entire Chicago population. Age Group 1, Age Group 2, and Age Group 3 refer to Infectious Class 1, Infectious Class 2, and Infectious Class 3 respectively.

According to the graph of the infectious subclasses, Age Group 2 (the green line) has the highest peak. The larger peak for Age Group 2 can be explained by the actual size of the age group, as Age Group 2 contains everyone between the ages of 18-59. Age Group 1 (the red line) and Age Group 3 (the blue line) both have peaks much lower. Notice that the size of the infectious class is greater for Age Group 1 than for Age Group 3. This graph shows that the eldest age group does not have as many cases as the other age groups. This variation between age groups is consistent with the data for Confirmed COVID-19 cases. For the actual collected data, there are more cases occurring with Age Group 2 than any other age group. Furthermore, there are more cases for Age Group 1 than for Age Group 3. It is interesting to note that the eldest age group has fewer cases yet contains the highest number of COVID-19 deaths. Comparison of COVID-19 mortality data with the model output is illustrated below.

The focus of this work was to incorporate the concept of frailty within an infectious disease model, where frailty affects death rate. Since the focus is to examine how 
accurately our method of frailty models the number of COVID-19 deaths per age group, we compare the mortality data collected with the model output for deaths more closely. The plots for the number of daily COVID-19 deaths and the number of cumulative deaths predicted by the model are illustrated below.

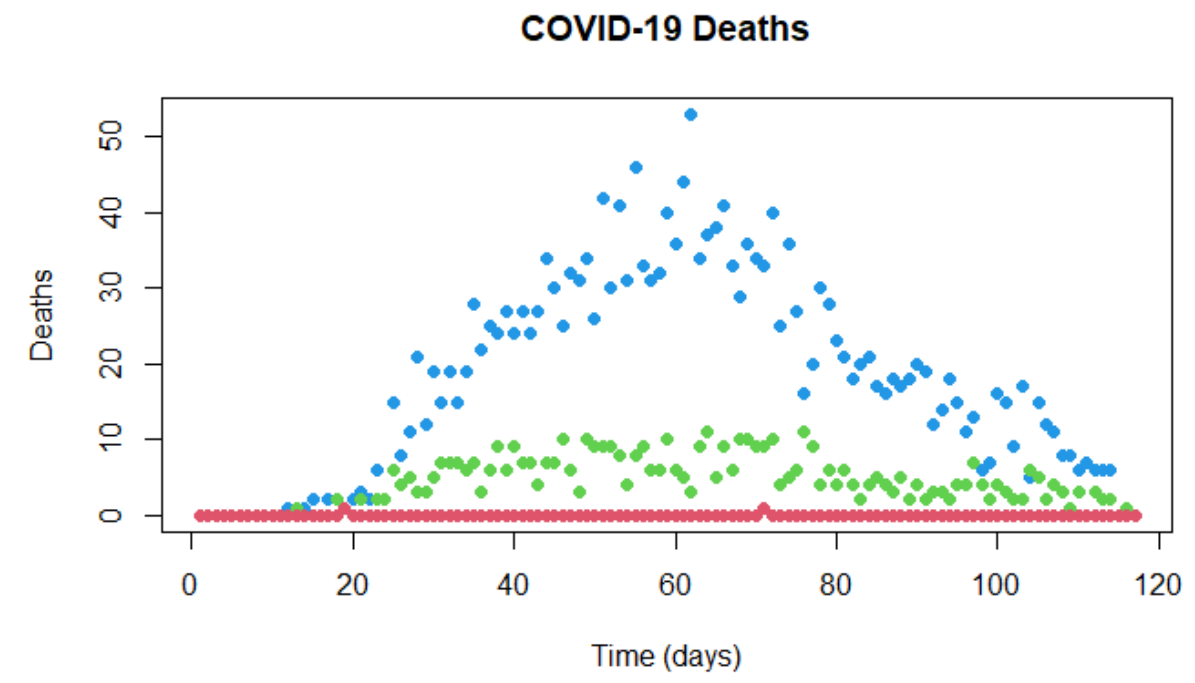

Figure 11: Number of daily COVID-19-induced deaths for each age group.

If frailty was not included within the model, the cumulative number of deaths for each age group is proportional to the number of cases within each age group, as illustrated below. 


\section{Cumulative Deaths}

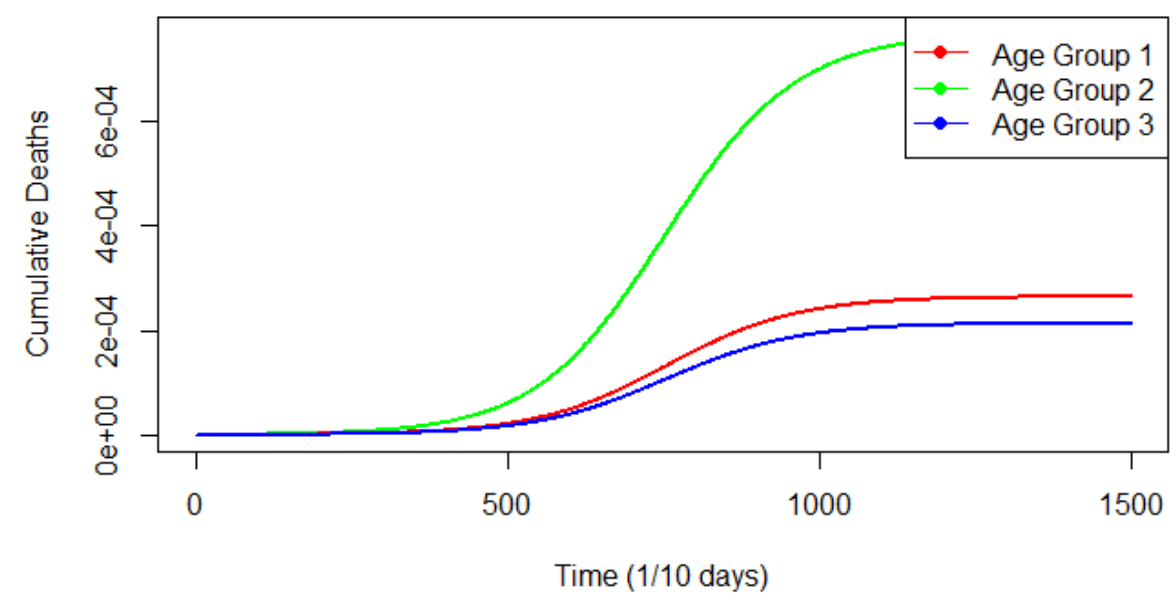

Figure 12: Cumulative COVID-19-induced deaths for each age group when no frailty parameters are included. Cumulative deaths are depicted as proportions of the entire Chicago population.

When frailty is accounted for, the cumulative number of deaths for each age group more closely reflects the actual data collected, as shown below.

\section{Cumulative Deaths}

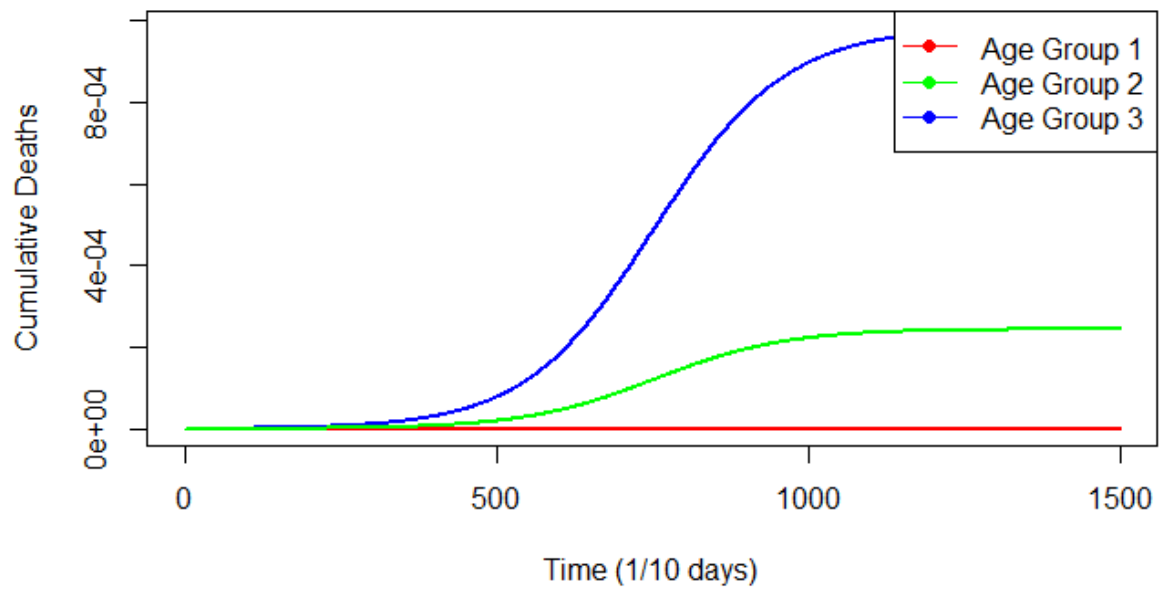

Figure 13: Cumulative COVID-19-induced deaths for each age group when frailty is accounted for. Cumulative deaths are depicted as proportions of the entire Chicago population. 
Using the frailty-inclusive model, the number of deaths predicted within Age Group 3 is higher than the number of deaths predicted in both Age Group 1 and Age Group 2 combined. This difference in predicted number of deaths between age groups is consistent with COVID-19 mortality data, where Age Group 3 has a couple thousand COVID-19-induced deaths, while Age Group 1 has at most two deaths and Age Group 2 has fewer than Age Group 3 but more than Age Group 1. Please note that the model graph of predicted deaths does not take the same shape as the plot of the raw mortality deaths. This is attributed to the fact that the model counts deaths cumulatively rather than as daily counts. The size of the D1, D2, and D3 subclasses changes over time in that each subclass increases most rapidly during the peak of the size of their corresponding infectious subclasses. The subclass D1 however, has very few deaths occur so this peak is difficult to examine visually. The subclass D2 grows at a faster rate than D1 but at a slower rate that D2. In this way, Age Group 2 does not reach a large cumulative number of COVID-19-induced deaths. Subclass D3 however, increases in size most rapidly at the start of the outbreak and throughout the peak. This rapid increase in the number of COVID-19-induced deaths within Age Group 3 is a result of the frailty parameter $\phi_{3}$ indicating a much larger death rate for the eldest age group. Therefore, Age Group 3 maintains the highest count for cumulative COVID-19-induced deaths. To compare the cumulative predicted number of COVID-19-induced deaths over the course of the outbreak with the current available data, we examine the percentage of error. The percentage of error gives a comparison of how closely the model's cumulative data reflects the actual number of deaths. For the actual number of deaths predicted over time, as well as the calculation for the percentage of error, see the Table (6) below.

Table 6: Percent Error for the Number of Deaths

\begin{tabular}{|c|c|c|c|c|}
\hline Age Group & Actual Deaths & Model Deaths & Absolute Error & Percent Error \\
\hline $0-17$ & 2 & 2.34 & 0.34 & 16.76 \\
$18-59$ & 514 & 599.17 & 85.17 & 16.57 \\
$60+$ & 2,118 & $2,416.48$ & 298.48 & 14.09 \\
\hline
\end{tabular}


Overall, the model predicts a greater number of deaths than the current data contains. This overestimation is attributed to the fact that the model predicts the total number of deaths over the entirety of the outbreak, and the outbreak in Chicago is still ongoing. Therefore, the model is forecasting age-specific deaths past the current data. Please note that Chicago appears to be nearing the end of the first outbreak and is experiencing fewer deaths each day. 


\section{CHAPTER V: CONCLUDING REMARKS}

In conclusion, this work focused on the concept of frailty and how variation in response to an infectious disease can impact typical infectious disease models. Frailty was incorporated as a multiplicative deviation differentiating age group death rates from the average disease-induced death rate. In this way, frailty was used to showcase how many more times an age-specific death rate differed from the overall average death rate. The 2020 COVID-19 outbreak in Chicago, Illinois was used as a case study to examine how well incorporated frailty parameters model mortality. While the model reflects the data well, the model does follow the assumption that contacts between individuals within a population were consistent from the very beginning to the very end of the outbreak. In actuality, these contact rates changed over time as the city of Chicago first implemented a lockdown and later began to slowly lift social distancing guidelines. These changes in guidelines are important to consider as the number of contacts affects the average expected number of new cases to arise from one singular case, known as the basic reproduction number. Within the model, the basic reproduction number is held constant, whereas realistically this number has changed over time. In this way, the model falls short by taking an averaged basic reproduction number and attributing it to the entire outbreak. Furthermore, our model is based on current data as the outbreak continues to take shape.

Data is continually being collected and as this data changes, our model theoretically would need to be adjusted to better reflect updated parameter estimations. As a result of data limitations, our model is dependent on the available data. Our model is also dependent on medical knowledge regarding the disease of interest - COVID-19. New research is continually being conducted to better understand COVID-19 and the parameters depend on the current understanding of how COVID-19 spreads. Two important parameter estimations stem from the length of the incubation period and infectious period for COVID-19. It is currently unknown whether these periods differ among age groups. As a result, it was assumed for the model that these periods were uniform across age groups. If 
these periods actually differ as a result of age, the model would further need to be updated. All in all, our parameter estimates and our frailty calculations are dependent on the development of our medical knowledge of COVID-19 and ongoing data collection. 


\section{REFERENCES}

[1] Getz WM, Salter R, Mgbara W. (2019) Adequacy of SEIR models when epidemics have spatial structure: Ebola in Sierra Leone. Phil. Trans. R. Soc. B 374: 20180282. http://dx.doi.org/10.1098/rstb.2018.0282

[2] (2020, June 26). Coronaviruses. National Foundation for Infectious Diseases. Retrieved from www.nfid.org/infectious-diseases/coronaviruses/

[3] Sauer, L.M. (2020, July 9th). What Is Coronavirus? Johns Hopkins Medicine. Retrieved from www.hopkinsmedicine.org/health/conditions-and-diseases/coronavirus

[4] Ruiz, L. A., España, P. P., Gómez, A., Bilbao, A., Jaca, C., Arámburu, A., Capelastegui, A., Restrepo, M. I., Zalacain, R. (2017). Age-related differences in management and outcomes in hospitalized healthy and well-functioning bacteremic pneumococcal pneumonia patients: a cohort study. BMC geriatrics, 17(1), 130. https://doi.org/10.1186/s12877-017-0518-0

[5] (2019). People Who Are at Increased Risk for Severe Illness. Center for Disease Control and Prevention. https://www.cdc.gov/coronavirus/2019-ncov/need-extraprecautions/people-at-higher-risk.html

[6] Valderas, J.M., Starfield, B., Sibbald, B., Salisbury, C., Roland, M. (2009, July). Defining Comorbidity: Implications for Understanding Health and Health Services. National Institute of Health.

https://www.ncbi.nlm.nih.gov/pmc/articles/PMC2713155/

[7] Guan W-j, Liang W-h, Zhao Y, et al. (2020). Comorbidity and its impact on 1590 patients with COVID-19 in China: a nationwide analysis. Eur Respir J 2020; 55: 2000547 https://doi.org/10.1183/ 13993003.00547-2020

[8] Divo, M. J., Martinez, C. H., Mannino, D. M. (2014). Ageing and the epidemiology of multimorbidity. The European respiratory journal, 44(4), 1055-1068. https://doi.org/10.1183/09031936.00059814 
[9] Lang, P. O., Michel, J. P., Zekry, D. (2009). Frailty syndrome: a transitional state in a dynamic process. Gerontology, 55(5), 539-549. https://doi.org/10.1159/000211949

[10] Clegg, A., Young, J., Iliffe, S., Rikkert, M. O., Rockwood, K. (2013). Frailty in elderly people. Lancet (London, England), 381(9868), 752-762. https://doi.org/10.1016/S0140-6736(12)62167-9

[11] De Gaudio AR, Rinaldi S, Chelazzi C, Borracci T. (2009). Pathophysiology of sepsis in the elderly: clinical impact and therapeutic considerations. Curr Drug Targets. 2009;10(1):60-70. doi:10.2174/138945009787122879

[12] Levy, J. (2020, June 25th). COVID-19 Daily Cases and Deaths. City of Chicago. https: / / healthdata.gov/dataset/covid-19-daily-cases-and-deaths

[13] Brauer, F., Castillo-Chavez, C. (2012). Mathematical Models in Populations Biology and Epidemiology. Texts in Applied Mathematics, 40, 345-409. https://link.springer.com/book/10.1007/978-1-4614-1686-9

[14] Mallapaty, S. (2020, June 16th). How deadly is the coronavirus? Scientists are close to an answer. Nature. https://www.nature.com/articles/d41586-020-01738-2

[15] Klein, J. (1992). Semiparametric Estimation of Random Effects Using the Cox Model Based on the EM Algorithm. Biometrics, 48(3), 795-806. doi:10.2307/2532345

[16] Hethcote HW. (2000). The mathematics of infectious diseases. SIAM Rev. 42, 599-653. (10.1137/S0036144500371907)

[17] Getz, W. M., Salter, R., Muellerklein, O., Yoon, H. S., Tallam, K. (2018). Modeling epidemics: A primer and Numerus Model Builder implementation. Epidemics, 25, 9-19. https://doi.org/10.1016/j.epidem.2018.06.001

[18] Keeling M. J. (1999). The effects of local spatial structure on epidemiological invasions. Proceedings. Biological sciences, 266(1421), 859-867. https://doi.org/10.1098/rspb.1999.0716 